\title{
On the Reusage of Primary Radio Resources through the Exploitation of the Spatiotemporal Robustness of the MIMO Transmissions
}

\author{
G. A. Medina-Acosta and José A. Delgado-Penín \\ Signal Theory and Communications Department, Technical University of Catalonia, Barcelona Tech, \\ Building D4 Campus Nord, Jordi Girona 31, 08034 Barcelona, Spain \\ Correspondence should be addressed to G. A. Medina-Acosta, agni_medina@tsc.upc.edu
}

Received 8 June 2012; Accepted 14 August 2012

Academic Editor: Ghazanfar Ali Safdar

Copyright (C 2012 G. A. Medina-Acosta and J. A. Delgado-Penín. This is an open access article distributed under the Creative Commons Attribution License, which permits unrestricted use, distribution, and reproduction in any medium, provided the original work is properly cited.

\begin{abstract}
The spatiotemporal robustness of the MIMO transmissions is exploited aiming at inducing opportunistic secondary communications on the primary radio resources. The proposed model makes use of the smallest singular values and the Extreme Value Theory for characterizing the robustness of the MIMO systems, having always the premise of preventing significant degradations in the performance of the primary communications, while at the same time an attractive number of potential opportunities for secondary are intended to be offered. So, the idea behind the suggested scheme consists in reusing the information that is already available within the primary MIMO networks aiming at cotransmitting opportunistically in a clever manner, which at the end will allow us to make a much more efficient use of both the natural and technical resources.
\end{abstract}

\section{Introduction}

After reviewing the vast activity related with the cognitive radio (CoRa) technology, it is quite easy to realize that the spectrum awareness turns out to be the keystone of this technology. This is because, according to the cognitive radio cycle [1], the first step for embedding cleverness to a radio transceiver has to do with the perception of the radio environment. In this regard, a methodology known as active spectrum awareness (i.e., spectrum sensing) has been widely studied for over ten years aiming at addressing this issue [26]. However until now it is still unclear which is (are) going to be the globally recommended technique(s) for carrying out this task. This because nowadays there are many scientific and technical issues (not expected to be solved in less than ten years) preventing that we be able to have a full CoRa device.

That is the reason why this research work proposes a model that is focused on making use of the advanced information that nowadays is inherently collected at the modern primary networks (e.g., multicarrier MIMO systems), aiming at establishing opportunistic secondary communications under an assisted detection scheme.
So, the LTE standard was selected as study object for the hypothesis made in this proposal, since its current releases and planned enhancements have inherently embedded an advanced network knowledge which could be reutilized in a clever manner by other technologies such as the cognitive radio.

Concretely, the information extracted from the channel state information in a single user MIMO LTE Uplink is reutilized aiming at establishing a CoRa communication over the particular time instants where the primary system is more resistant to the impairments. This is done by developing a model which pretends to identify the spatiotemporal robustness of the MIMO system by making use of the smallest singular values and the extreme value theory. So, as part of the obtained results several thresholds were determined aiming at evaluating the disruptions caused on the performance of the primary user (MIMO LTE uplink) as a function of the number of discovered opportunities for secondary purposes.

This paper is organized as follows. Section 2 provides a brief review of the MIMO systems, to later on 
introducing the implemented MIMO SC-FDMA or LTEA uplink. Section 3 describes the proposed opportunistic CoRa communication model which works over primary MIMO transmissions. Section 4 shows and discusses in detail the obtained results. Finally, Section 5 summarizes the conclusions and provides an insight about the future work.

\section{The MIMO Systems and the LTE-A Uplink}

2.1. The MIMO Systems. Instead of transmitting data over a single radio channel, a multiple-input-multiple-output (MIMO) system transmits over several radio channels aiming at increasing the data rate (spatial multiplexing) or improving the BER (transmit diversity) [7-9]. As an illustrative example, let us assume a MIMO system that is composed by four transmit antennas (i.e., $A_{1}, A_{2}, A_{3}$, and $\left.A_{4}\right)$ and four receive antennas (i.e., $B_{1}, B_{2}, B_{3}$, and $B_{4}$ ). Such a system transmits simultaneously through all its available antennas, which originates that at the receiver every single antenna will be a witness of signals coming from $A_{1}, A_{2}$, $A_{3}$, and $A_{4}$, respectively. In fact, the relationship between the received and transmitted signals can be written as

$$
\mathbf{B}(t)=\mathbf{H}(t) \mathbf{A}(t) .
$$

In the above equation $\mathbf{H}$ refers to the channel transfer function matrix, which corresponds to a certain time instant, as well as to a particular spatial location of the antennas. Concretely, whenever the received signals are sufficiently independent, a MIMO structure has the potential of increasing the capacity by the amount of the minimum number of antennas embedded in the system (i.e., by four in the case of the above-cited example when spatial multiplexing is considered) with respect to a conventional SISO system. Highlighting that the desired independence degree is close related to the propagation scenario in question, that means that a rich multipath environment theoretically will lead to uncorrelated signals at the receiver, while line-of-sight conditions simply would break the principle behind a MIMO transmission (i.e., LOS leads to a high correlation in the received signals).

Mathematically speaking, the algebraic rank $R$ of the channel matrix dictates the number of independent equations provided by the MIMO system, which in practice (even inside a rich multipath environment) could be decreased by the presence of other propagation effects such as diffraction and wave guiding. However, and roughly speaking, the fact of embedding MIMO arrays to the wireless communications systems brings a significant improvement to the system's performance, since the artificially created channels by the multiple antennas rarely are going to experience deep fadings simultaneously (i.e., the information is expected to be properly recovered after combining what is received at the set of the reception antennas).

2.2. The MIMO SC-FDMA (LTE-A Uplink). The baseband block diagram of the MIMO SC-FDMA transceiver chain as used by LTE-A is shown in Figure 1.
The above figure depicts the transmitter, as well as the receiver structure of a $2 \times 2$ spatially-multiplexed MIMO SC-FDMA system (Closed Loop). At the transmitter, the procedure initiates in the time domain with the serial arranged symbols which in first instance are demuxed aiming at building two codewords (CW), then each of them are arranged in parallel in order to be modulated and fed to an $N$-point fast Fourier transform (FFT) which produces a set of modified complex numbers in the frequency domain. At this point the information has been spread out over the $N$ subcarriers, subsequently a procedure per subcarrier initiates in order to carry out a CW-to-layer mapping (the number of layers enabled depends on the rank of the MIMO channel), which precedes the precoding block (a precoding matrix is utilized by a signal processing technique known as beamforming, which aims at combining the layers before transmission for equalizing the signal reception). So, the output of the so-called per subcarrier procedure can be expressed as

$$
\mathbf{q}[k]=\mathbf{F}[k] \mathbf{x}[k]
$$

where $\mathbf{F}[k]$ refers to the precoding matrix (a simple Rank2 precoding matrix as suggested by LTE-A maps each layer to a single antenna without combining the layers: $\mathbf{F}[k]=$ $1 / \sqrt{2}\left[\begin{array}{ll}1 & 0 \\ 0 & 1\end{array}\right]$ ), and $\mathbf{x}[k]$ to a vector (i.e., with length equal to the rank) that has to do with the output of the CW-to-layer mapping. Then per every layer the procedure is continued by mapping one-on-one the precoded signals over a set of $N$ contiguous subcarriers (zeros are placed into the $M$ $N$ unused subcarriers), just after the $M$-point inverse fast Fourier transform (IFFT) is applied for transforming the information to the time domain, which is set back into a serial form for adding a cyclic prefix, letting this way the baseband SC-FDMA symbol ready to be transmitted through the channel [10-12].

Once a circular convolution per channel took place (e.g., theoretically there are four virtual links in this array) the set of initial steps per layer at the receiver consists in removing the cyclic prefix, putting the information into parallel form, and utilizing the $M$-point FFT to transform back the received information to the frequency domain, and later on a demapping procedure is carried out aiming at strictly preserving the $N$ subcarriers allocated to that user, which are equalized on a per subcarrier procedure in order to provide a better estimation of the originally modified complex numbers. The output of the embedded MMSE equalizer (i.e., by assuming perfect channel knowledge) for the $k$ th subcarrier can be written as [13]

$$
\begin{aligned}
\mathbf{y}_{\mathrm{eq}}[k]= & \left(\mathbf{F}[k]{ }^{H} \mathbf{H}[k]{ }^{H} \mathbf{H}[k] \mathbf{F}[k]+\sigma_{w}^{2} \mathbf{I}_{R}\right)^{-1} \\
& \times\left(\mathbf{F}[k]^{H} \mathbf{H}[k]{ }^{H} \mathbf{y}[k]\right) .
\end{aligned}
$$

In the above equation, $\sigma^{2}$ refers to the variance of the additive white Gaussian noise, $\mathbf{I}_{R}$ has to do with an identity 


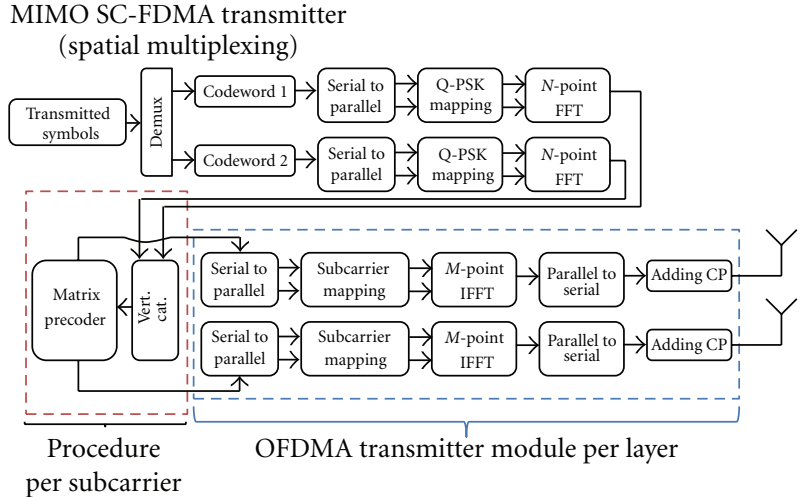

(a)



(b)

FIgURE 1: Transmitter and receiver structure of a $2 \times 2$ MIMO SCFDMA system.

matrix of size $R \times R$, while the transfer function of the channel matrix is described as

$$
\mathbf{H}[k]=\left[\begin{array}{ccc}
h_{11}(k) & \cdots & h_{1 N_{T}}(k) \\
\vdots & \ddots & \vdots \\
h_{N_{R} 1}(k) & \cdots & h_{N_{R} N_{T}}(k)
\end{array}\right]
$$

In (4), the notation $h_{i j}(k)$ refers to the complex channel gain from the $T_{x}$ antenna $j$ to the $R_{x}$ antenna $i$, being $N_{T}$ and $N_{R}$ the total number of $T_{x}$ and $R_{x}$ antennas, respectively.

Then, continuing with the description of the procedure performed at the receiver, the best estimation of the modified complex numbers is arranged in parallel (i.e., per CW) to be later on transformed back to the time domain aiming at obtaining the input needed by the demodulator, which provides the recovered symbols that are finally feed to a MUX and rearranged in serial form.

In the SC-FDMA system a generic radio frame has a duration of $10 \mathrm{~ms}$ and consists of 20 slots. On the other hand, a subframe is composed by 2 slots each slot, being built from seven or six SC-FDMA symbols depending on the cyclic prefix length configuration (i.e., normal or extended). So, based on the above, it is possible to write an equation describing the way of computing the peak bit rates of the system (i.e., per every branch of the MIMO structure) [14]:

Peak bit rate $=\frac{(\text { bits })(\text { subcarriers })(\text { symbols per subframe })}{1 \mathrm{~ms}}$.

In the numerator, starting from left to right we have the number of bits given by the modulation in use (e.g., Q-PSK will lead to 2 bits), then the subcarriers are related with those that were allocated to the user in question, while the number of symbols per subframe depends on the cyclic prefix length just as it was mentioned before. For its part, the denominator refers to the required time for transmitting a subframe.

\section{An Opportunistic Cognitive Radio Communication Model over MIMO Transmissions}

3.1. The Role of the Smallest Singular Values. Under the context of a MIMO transmission in the LTE uplink, we refer to the particular case of having two transmit antennas and two receive antennas, just as it was described in Section 2.2.

We know that after decomposing the channel matrix $\mathbf{H}$ (e.g., by using SVD), it is possible to obtain a matrix $\Sigma$ of singular values $\lambda_{i}$ :

$$
\Sigma=\left[\begin{array}{cc}
\lambda_{1} & 0 \\
0 & \lambda_{2}
\end{array}\right]=\left[\begin{array}{cc}
\lambda_{1} & 0 \\
0 & \lambda_{\min }
\end{array}\right]
$$

highlighting that every transmitted subcarrier undergoes the impairments given by all the channels involved, and hence each of them has associated a matrix of singular values like the one shown above. In this regard, we know from matrix algebra that for any vector $x$ the following holds [15]

$$
\|H x\| \geq \lambda_{\min }\|x\|,
$$

where $\lambda_{\min }$ is the smallest singular value of $\mathbf{H}$. If $x$ is considered as the difference between the possible transmitted symbols (i.e., $x=s_{i}-s_{j}$, being $i \neq j$ ): we have

$$
\left\|H\left(s_{i}-s_{j}\right)\right\| \geq \lambda_{\min }\left\|s_{i}-s_{j}\right\| .
$$

For a symbol error to occur, the norm of the noise vector would have to go beyond half the minimum distance:

$$
\|n\| \geq \frac{\left(\min _{i \neq j}\left\|H\left(s_{i}-s_{j}\right)\right\|\right)}{2} \quad \text { or } \quad\|n\| \geq \frac{d_{\min }}{2} .
$$

But (8) tell us that a large value of $\lambda_{\text {min }}$ guarantees a large value of $d_{\text {min }}$, leading hence to a lower probability of error. So, our hypothesis is that channel matrices having the best smallest singular values (i.e., the largest ones) in a MIMO transmission could be identified in order to superpose an opportunistic secondary communication without degrading in a significant manner the performance of the primary system. So, our objective is to put our attention into the largest singular values which arise in between the smallest singular values in a MIMO transmission. 
3.2. The Application of the Extreme Value Theory. In a MIMO transmission, the sudden arising of the largest singular values in between the smallest ones leads to the most robust four-dimensional combination (i.e., latitude, longitude, frequency, time) for facing better the errors in a communication. Therefore by using the Extreme Value Theory we have modeled the arising of such largest singular values aiming at studying the feasibility of opportunistically interfering (i.e., with a superimposed CoRa communication) a LTE-MIMO transmission without significantly degrading its performance, while at the same time an attractive number of potential opportunities for a secondary transmission are being offered.

According to the Central Limit Theorem, any population or parent distribution having finite variance will lead to the following fulfillment: the probability density function (PDF) of the means coming from $n$ observations (consisting each of a block or number of successive independent and identically distributed random variables) will get closer and closer to a normal distribution as $n$ increases.

So, once the Central Limit Theorem has been cited, then it is possible to introduce an analogous result which applies for the extreme values instead of the means, leading this time to a PDF having the following form [16] ( highlighting that the variable $x$ is shown in (10) and is different to the one utilized in (7))

$$
\begin{array}{r}
H(x ; K, \sigma, \mu)=\exp \left[-\left(1+K \frac{x-\mu}{\sigma}\right)^{-1 / K}\right], \\
\text { for } 1+K \frac{x-\mu}{\sigma}>0 .
\end{array}
$$

The above equation is known as the Generalized Extreme Value (GEV) distribution and encompasses the Gumbel (Type I), Frechet (Type II), and Weibull (Type III) distributions. In general, for some values of $K$ (shape parameter), $\sigma$ (scale parameter), and $\mu$ (location parameter) the distribution of the maximums (or minimums) must take the form of the GEV distribution, which is classified as Type III when $K<0$, as Type II when $K>0$, and as Type I as $K$ approaches to 0 . One way of estimating the abovementioned parameters is by using the maximum likelihood method, which will provide us with the required parameters maximizing the good modeling of the observed data set. On the other hand, beyond of fitting the extreme values coming from an unknown parent distribution to a GEV distribution, this theory can also provide an insight about the maximum/minimum values that may occur in the future. The so-called return level $\left(R_{m}\right)$ can be used for this purpose, and in a broad sense refers to a certain level that is expected to be exceeded once in $m$ blocks, which can be computed as follows:

$$
R_{m}=F^{-1}\left(1-\frac{1}{m}\right),
$$

where $F^{-1}$ is the inverse cumulative distribution function (CDF), which is generated from the previously obtained GEV distribution. Nevertheless, in spite of being able to compute $R_{m}$ by using the above equation, it has to be considered that

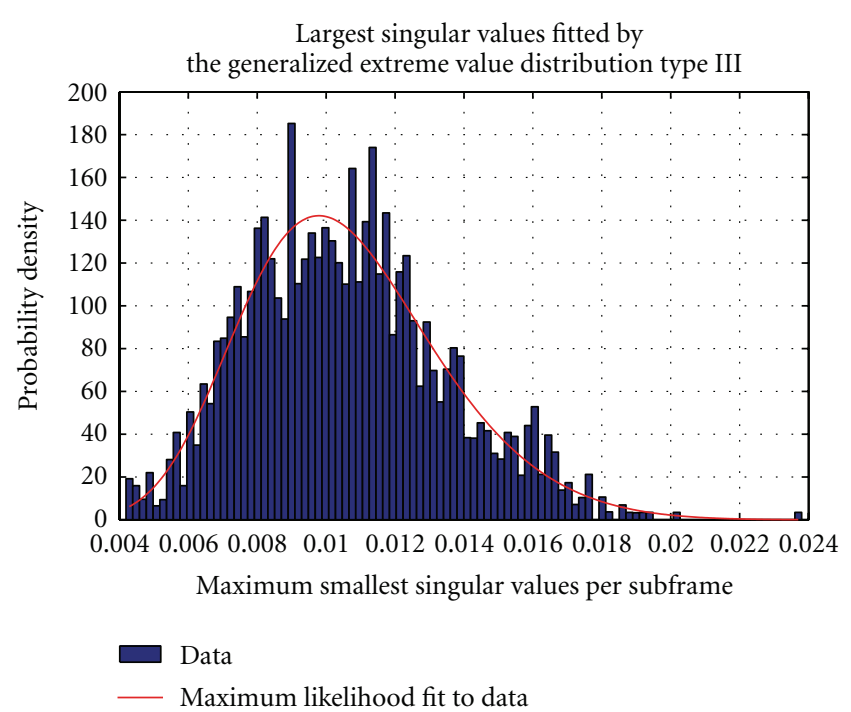

FIGURE 2: Fitted PDF of the largest singular values on a per subframe basis.

this one does not have a perfect accuracy since the parameters (i.e., $K, \sigma$, and $\mu$ ) leading to $F^{-1}$ are not perfect either. This is indeed the reason why we should look for a region where the estimated parameters lead to distributions that are always consistent with the data set in question, being the final aim to find the limits for the value of $R_{m}$, which is the variable that we are trying to forecast.

So, instead of estimating a unique set of MLEs for the parameters $K, \sigma$, and $\mu$, it is also possible to estimate several sets of parameters in such a way that the GEV loglikelihood is above a specified critical value (a suitable one is based on a chi-square approximation) and therefore remains maximized according to the data set in question. So, after identifying the parameters describing a region where the loglikelihood (or negative log-likelihood) results to be larger than the selected critical value, then it is possible to compute $R_{m}$ for all the cases involved.

\section{Performance Evaluation of the MIMO Transmission Subject to Low Impact Interference}

The parameters implicitly involved into the implementation of a single user MIMO SC-FDMA LTE $(2 \times 2$ spatial multiplexing scheme) are summarized in Table 1 [17-20].

In order to study the sudden arising of the largest singular values in between the smallest ones, 11,200,000 samples were collected. So, aiming at extracting the maximums, blocks of data were created on per-subframe basis in such a way that at the end 25,000 maximums were obtained.

The GEV distribution fitting the data set representing the largest singular values is shown in Figure 2.

In the above figure, the histogram shown was scaled in order to make it comparable to the fitted PDF, which was obtained as described in Section 3.2. In a complementary 
TABLE 1: Simulation parameters of the MIMO SC-FDMA (LTE-A Uplink).

\begin{tabular}{lcl}
\hline Variable & Value & Description \\
\hline$p$ & 128 & Total number of subcarriers in the system \\
$k$ & 32 & Number of subcarriers per user \\
$q$ & 4 & $\begin{array}{l}\text { Number of simultaneous transmissions (users) without cochannel } \\
\text { interference } \\
\text { Channel bandwidth (MHz) }\end{array}$ \\
BW & 1.4 & Sampling time (us) \\
$T_{s}$ & 0.52083 & Carrier frequency (MHz) \\
$f_{c}$ & 1850.7 & Number of codewords \\
$\mathrm{CW}$ & 2 & Algebraic rank (enabled layers) \\
$R$ & 2 & \\
$\mathbf{F}[k]$ & 1 & Precoding matrix (kth subcarrier) \\
$\mathbf{Y}_{\text {eq }}[k]$ & $\left(\mathbf{F}[k]^{H} \mathbf{H}[k]^{H} \mathbf{H}[k] \mathbf{F}[k]+\sigma_{w}^{2} \mathbf{I}_{R}\right)^{-1}\left(\mathbf{F}[k]^{H} \mathbf{H}[k]^{H} \mathbf{y}[k]\right)$ & MMSE equalizer (frequency domain, see (3) Section 2 for more details) \\
\hline
\end{tabular}



Figure 3: Fitted CDF of the largest singular values on a per subframe basis.

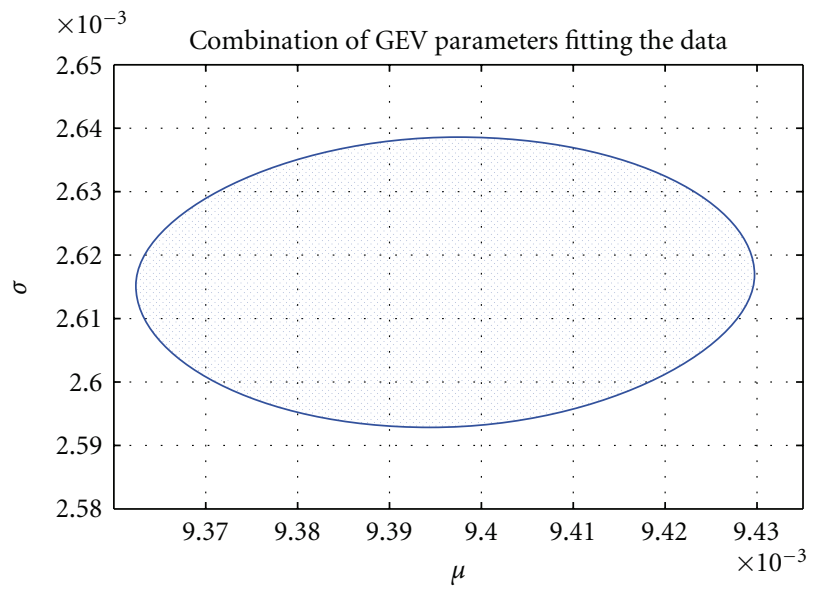

FIGURE 4: Region of GEV parameters leading to a PDF modeling the data set.

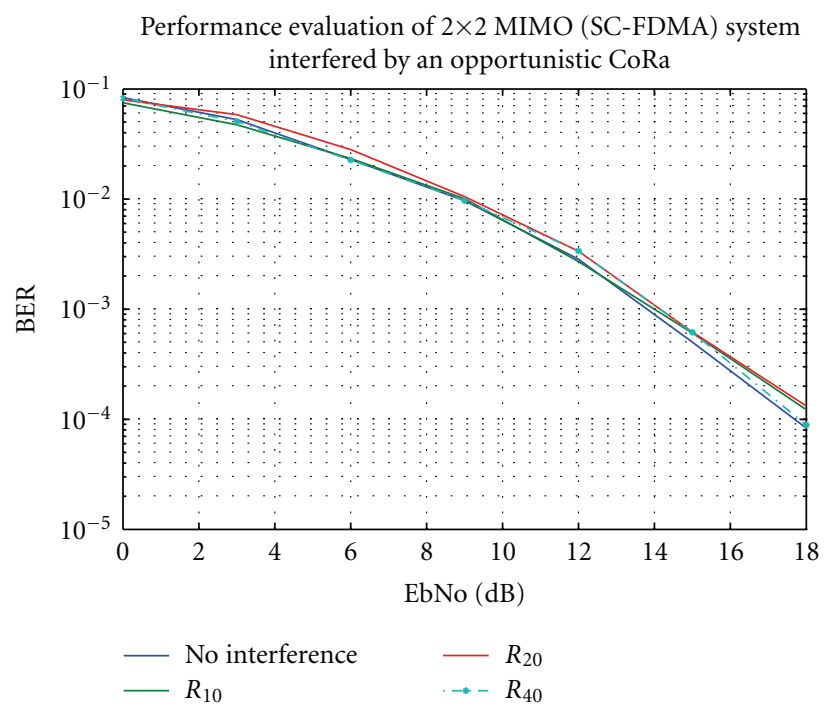

FIGURE 5: BER of the interfered primary user (MIMO SC-FDMA).

way, the CDF was determined as can be appreciated in Figure 3.

On the other hand, by fixing the value of the $K$ parameter, a region containing a family of parameters also fitting the data set is shown in Figure 4.

So, a particular return of level $R_{m}$ was computed for every point described by the above ellipsoid, being the maximum and the minimum values within the region the ones utilized as a threshold for enabling the secondary access (i.e., when in a primary transmission a minimum singular value be found matching the range given by the threshold, then the secondary access on a CoRa OFDMA basis is granted).

This way and by repeating the above procedure, several return levels were forecasted aiming at testing their impact on the primary communication, just as shown in Figure 5. 
TABLE 2: Theoretical peak bit rates (PU and SU).

\begin{tabular}{lccc}
\hline User type & Return of level & $\begin{array}{c}\text { Data rate } \\
(\mathrm{Q}-\mathrm{PSK})\end{array}$ & $\begin{array}{c}\text { Data rate } \\
(64 \mathrm{QAM})\end{array}$ \\
\hline $\mathrm{PU}$ & - & $896 \mathrm{Kbps}$ & $2.688 \mathrm{Mbps}$ \\
$\mathrm{SU}$ & $R_{5}$ & $12.8 \mathrm{Kbps}$ & $38.4 \mathrm{Kbps}$ \\
$\mathrm{SU}$ & $R_{10}$ & $6.4 \mathrm{Kbps}$ & $19.2 \mathrm{Kbps}$ \\
$\mathrm{SU}$ & $R_{20}$ & $3.2 \mathrm{Kbps}$ & $9.6 \mathrm{Kbps}$ \\
$\mathrm{SU}$ & $R_{30}$ & $2.1 \mathrm{Kbps}$ & $6.4 \mathrm{Kbps}$ \\
$\mathrm{SU}$ & $R_{40}$ & $1.6 \mathrm{Kbps}$ & $4.8 \mathrm{Kbps}$ \\
\hline
\end{tabular}

In general, from the above figure it can be noticed that when the primary system was interfered by the opportunistic secondary communication, the system's performance did not move too much away from the reference curve. In fact, for the carried-out analysis the distortion never went beyond one order of magnitude since for all cases the BER was maintained around $10^{-4}$, while the undistorted system kept its BER towards $10^{-5}$ (to remember that no error correction codes were implemented, if they were added probably the induced distortion would be less significant). On the other hand, the total number of discovered opportunities for the secondary user is indeed given by the threshold in use. That is, (theoretically speaking) a return of level $R_{10}$ will allow to the secondary user cotransmitting along with a primary SC-FDMA symbol once per every ten subframes. So, less restrictive return of levels will lead to more opportunities for the CoRa user. In this regard, the achieved throughputs by the $S U$ can be concluded by modifying (5) as a function of the return of level (i.e., the denominator changes as a function of the access granted). The maximum theoretical throughput by the PU, as well as those obtained by the SU with the input parameters used in the earlier simulations, are shown in Table 2.

On the other hand, if as instead of considering only $k=32$ as useful subcarriers, the maximum number of usable subcarriers (i.e., 72, 180, 300, 600, 900, and 1200) per bandwidth configuration in LTE (i.e., $1.4 \mathrm{MHz}, 3 \mathrm{Mhz}$, $5 \mathrm{Mhz}, 15 \mathrm{Mhz}$, and $20 \mathrm{Mhz}$ ) considered, then a primary user using a Q-PSK modulation would be able to achieve the following data rates: $2.016 \mathrm{Mbps}, 5.04 \mathrm{Mbps}, 8.4 \mathrm{Mbps}$, 16.8 Mbps, 25.2 Mbps, and 33.6 Mbps, respectively, while the obtained ones by the SU would be given according to those depicted in Figure 6.

As it can be verified from the above set of curves, most of the obtained data rates by the SU would be better described in terms of Kbps $\left(R_{1}:\{114 \mathrm{Kbps}, 360 \mathrm{Kbps}\right.$, $600 \mathrm{Kbps}, 1200 \mathrm{Kbps}, 1800 \mathrm{Kbps}, 2400 \mathrm{Kbps}\}, \mathrm{R}_{5}:\{28.8 \mathrm{Kbps}$, $72 \mathrm{Kbps}, 120 \mathrm{Kbps}, 240 \mathrm{Kbps}, 360 \mathrm{Kbps}, 480 \mathrm{Kbps}\}, R_{10}$ : \{14.4 Kbps， $36 \mathrm{Kbps}, 60 \mathrm{Kbps} ， 120 \mathrm{Kbps} ， 180 \mathrm{Kbps}, 240$ $\mathrm{Kbps}\}, R_{20}:\{7.2 \mathrm{Kbps}, 18 \mathrm{Kbps}, 30 \mathrm{Kbps}, 60 \mathrm{Kbps}, 90 \mathrm{Kbps}$, $120 \mathrm{Kbps}\}, \quad R_{30}:\{4.8 \mathrm{Kbps}, 12 \mathrm{Kbps}, 20 \mathrm{Kbps}, 40 \mathrm{Kbps}$, $60 \mathrm{Kbps}, 80 \mathrm{Kbps}\}, R_{40}:\{3.6 \mathrm{Kbps}, 9 \mathrm{Kbps}, 15 \mathrm{Kbps}, 30 \mathrm{Kbps}$, $45 \mathrm{Kbps}, 60 \mathrm{Kbps}\})$. At this point, it could be thought that the expected data rates by the SU result to be very slow; however, we have to keep in mind that we are dealing with a low priority communication, and if we take a look at

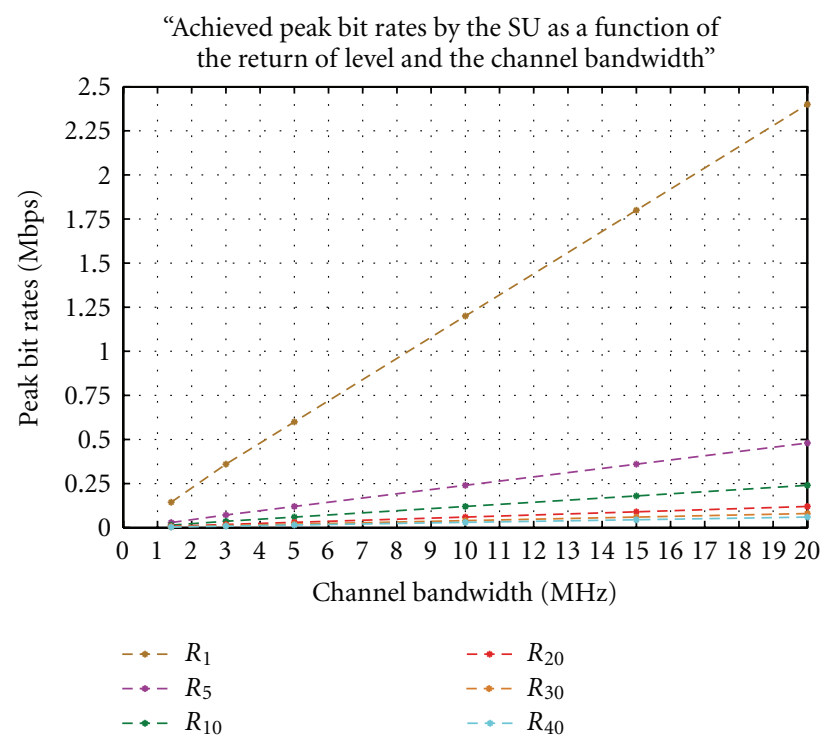

FIGURE 6: Peak data rates of a SU cotransmitting opportunistically along with a PU in an LTE system.

the past we would realize that in the GSM only rates up to $9.6 \mathrm{Kbps}$ were possible at that moment. Hence at the very beginning, the CoRa applications should be oriented to nonreal time tasks. In this regard, and talking about the achievable transmission speeds by the SUs, different secondary data rates have been reported in literature for MIMO systems being interfered by cognitive radios [21-24], which are difficult to be compared on a one-to-one basis, this since each proposed model has its own strategy for granting the secondary access (e.g., the model in [24] tracks the idle slots, while the one in [21] proposes a precoding scheme for avoiding to interfere the primary system).

On the other hand and getting back to our proposal, although the arising of the singular values of interest is well characterized, a much more realistic scenario should encompass the time varying nature of the mobile channels which could lead to a severe interference of the primary system. For that reason, in the future this proposal should be extended by considering such a small scale fading mechanism, which among other things will allow us to acquire the necessary knowledge for determining the equations corresponding to probability of collision detection and false alarm of the proposed scheme. Therefore, for the moment, this research work should be seen as a framework connecting the CoRa technology and the MIMO systems waiting to be complemented in order to increase the feasibility of its implementation.

\section{Conclusions}

In this research work, the extreme value theory was utilized for modeling the tracking of the most robust singular values appearing on the MIMO matrices, which represent to be the spatiotemporal conditions that are more resistant to the impairments within a MIMO system. 
So the proposed model was focused on guaranteeing the nonsevere disturbance of a primary MIMO user, while an attractive number of potential opportunities for secondary purposes were also pursued. In this study, several thresholds (return levels) were obtained from the proposed model, which were tested over the MIMO SC-FDMA spatial multiplexing transmission (3 GPP-LTE/LTE-A uplink) which played the role of the primary system. From the conduced analysis it was found that the performance degradation of the primary user never went beyond one order of magnitude with respect to an uninterfered communication used as reference, while the total number of secondary opportunities was given by the own selected threshold (i.e., by tuning $R_{m}$, the distortion degree undergone by the $\mathrm{PU}$ can be controlled). On the other hand, in addition to the fact of analyzing deeply the small-scale fading mechanisms on the proposed model, since this analysis was focused on studing the degradation suffered by the primary transmission once a low impact interference was induced to the system, the performance analysis of the secondary user remains also open as a future work, the successive cancellation being a suitable technique for achieving the required secondary BER.

\section{Acknowledgments}

The authors would like to thank the anonymous reviewers for their helpful comments, the COST Action IC0905 TERRA (because part of this work was presented there), and to the Technical University of Catalonia (UPC) for the financial support granted.

\section{References}

[1] I. F. Akyildiz, W. Y. Lee, and K. R. Chowdhury, "CRAHNs: cognitive radio ad hoc networks," Ad Hoc Networks, vol. 7, no. 5, pp. 810-836, 2009.

[2] S. Haykin, "Cognitive radio: brain-empowered wireless communications," IEEE Journal on Selected Areas in Communications, vol. 23, no. 2, pp. 201-220, 2005.

[3] D. Cabric, "Addressing the feasibility of cognitive radios: using testbed implementation and experiments for exploration and demonstration," IEEE Signal Processing Magazine, vol. 25, no. 6, pp. 85-93, 2008.

[4] T. Yücek and H. Arslan, "A survey of spectrum sensing algorithms for cognitive radio applications," IEEE Communications Surveys and Tutorials, vol. 11, no. 1, pp. 116-130, 2009.

[5] A. Ghasemi and E. S. Sousa, "Spectrum sensing in cognitive radio networks: requirements, challenges and design tradeoffs," IEEE Communications Magazine, vol. 46, no. 4, pp. 3239, 2008.

[6] Q. Zhao and B. M. Sadler, "A survey of dynamic spectrum access," IEEE Signal Processing Magazine, vol. 24, no. 3, pp. 7989, 2007.

[7] L. Zheng and D. N. C. Tse, "Diversity and multiplexing: a fundamental tradeoff in multiple-antenna channels," IEEE Transactions on Information Theory, vol. 49, no. 5, pp. 10731096, 2003.

[8] S. Sanayei and A. Nosratinia, "Antenna selection in MIMO systems," IEEE Communications Magazine, vol. 42, no. 10, pp. 68-73, 2004.
[9] D. Gesbert and J. Akhtar, "Breaking the barriers of Shannon's capacity: an overview of MIMO wireless systems," Telektron$i k k$, vol. 98, no. 1, pp. 53-64, 2002.

[10] H. Holma and A. Toskala, LTE For UMTS OFDMA and SCFDMA Based Radio Acces, John Wiley \& Sons, Chichester, UK, 2009.

[11] H. G. Myung, "Introduction to Single Carrier FDMA," in Proceedings of the 15th European Signal Processing Conference (EUSIPCO '07), Poznan, Poland, September 2007.

[12] H. G. Myung, Single carrier orthogonal multiple access technique for broadband wireless communications [Ph.D. dissertation], Department of Electrical and Computer, Polytechnic University, Brooklyn, NY, USA, 2007.

[13] G. Berardinelli, T. B. Sørensen, P. Mogensen, and K. Pajukoski, "SVD-based vs. Release 8 codebooks for single user MIMO LTE-A uplink," in Proceedings of the IEEE 71st Vehicular Technology Conference (VTC '10), pp. 1-5, May 2010.

[14] P. Wang, "LTE Physical Layer Overview," 2011, http://wwwee.uta.edu/Online/PWang/ee5368/Spring2011/Review\%20_2_ LTE\%20Physical\%20Layer\%20Overview_01162011.pdf.

[15] G. Burel, "Statistical analysis of the smallest singular value in MIMO transmission system," in Proceedings of the International Conference on Signal, Speech, and Image Processing (WSEAS '02), Skiathos Island, Greece, September 2002.

[16] E. Castillo, Extreme Value Theory in Engineering, Academic Press, 1998.

[17] 3GPP TS 36.101 V9.4.0, "User Equipment (UE) radio transmission and reception," 2010.

[18] 3GPP TR 25.814 V7.1.0, "Physical Layer aspects for evolved Universal Terrestrial Radio Access (UTRA)," 2006.

[19] 3GPP TS 36.211 V9.1.0, "Physical Channels and Modulation," 2010.

[20] 3GPP TR 25.943 V9.0.0, “Technical Specification Group Radio Access Network: Deployment aspects," 2009.

[21] Z. Chen, C.-X. Wang, X. Hong et al., "Interference Mitigation for cognitive radio MIMO systems based on practical precoding," to appear in IEEE Transactions on Vehicular Technology, http://arxiv.org/abs/1104.4155v1.

[22] K. Hamdi, W. Zhang, and K. B. Letaief, "Opportunistic spectrum sharing in cognitive MIMO wireless networks," IEEE Transactions on Wireless Communications, vol. 8, no. 8, pp. 4098-4109, 2009.

[23] R. Zhang and Y. C. Liang, "Exploiting multi-antennas for opportunistic spectrum sharing in cognitive radio networks," IEEE Journal on Selected Topics in Signal Processing, vol. 2, no. 1, pp. 88-102, 2008.

[24] H. Huang, Z. Zhang, P. Cheng, G. Yu, and O. Qui, “Throughput analysis of cognitive MIMO system," in Proceedings of the International Workshop on Cross Layer Design (IWCLD '07), Jinan, China, September2007. 

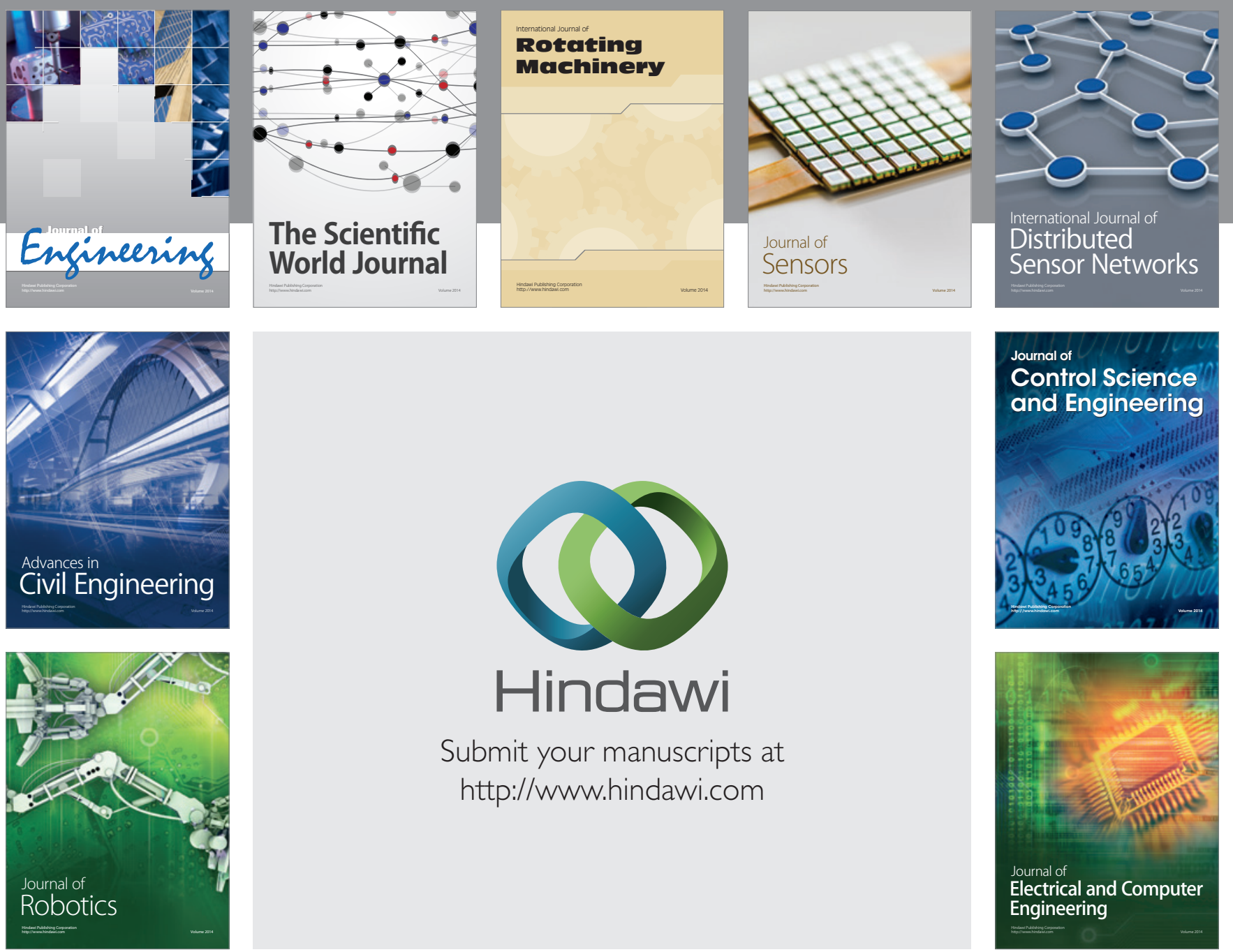

Submit your manuscripts at

http://www.hindawi.com
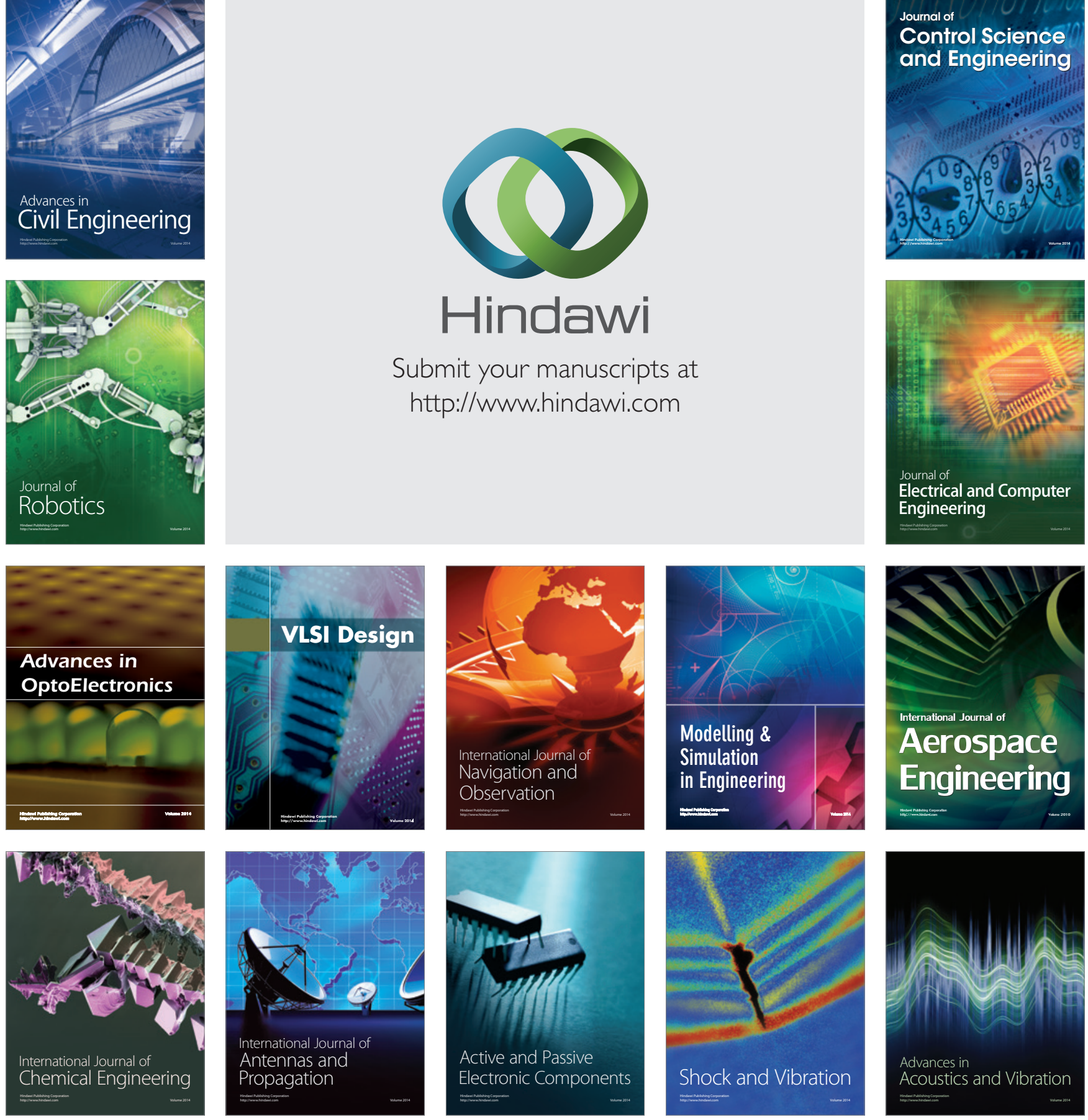